\title{
GENERALISATIONS OF NILPOTENCY IN RINGS
}

\author{
by EDMUND F. ROBERTSON
}

(Received 27th May 1972)

In (5) and (6) we studied certain subgroups of infinite dimensional linear groups over rings. In particular we investigated how the structure of the subgroups was related to the structure of the rings over which the linear groups were defined. It became clear that it might prove useful to study generalised nilpotent properties of rings analogous to Baer nilgroups and Gruenberg groups. We look briefly at some classes of generalised nilpotent rings in this paper and obtain a lattice diagram exhibiting all the strict inclusions between the classes.

\section{Notation}

We shall use the notation $\mathscr{A}$ for the class of rings with zero multiplication and $\mathcal{N}$ for the class of nilpotent rings. The class of rings which are the sum of their nilpotent ideals we shall denote by $\mathscr{F}$.

A subring $S$ of a ring $R$ is a subideal of $R$ if there exist subrings $S_{0}, S_{1}, S_{2}$, $\ldots, S_{n}$ of $R$ with

$$
S=S_{0} \subseteq S_{1} \subseteq S_{2} \subseteq \ldots \subseteq S_{n}=R,
$$

where $S_{i-1}$ is an ideal of $S_{i}, 1 \leqq i \leqq n$.

Notice that in a nilpotent ring every subring is a subideal. For, if $R \in \mathscr{N}$ then $R^{n}=(0)$ for some $n \geqq 1$. Put $S_{i}=S+R^{i}$. Then

$$
S=S_{n} \subseteq S_{n-1} \subseteq \ldots \subseteq S_{1}=R,
$$

where $S_{i}$ is an ideal of $S_{i-1}$. Since, if $s_{1}+r_{i} \in S+R^{i}$ and $s_{2}+r_{i-1} \in S+R^{i-1}$ then

$$
\left(s_{1}+r_{i}\right)\left(s_{2}+r_{i-1}\right)=s_{1} s_{2}+\left(r_{i} s_{2}+s_{1} r_{i-1}+r_{i} r_{i-1}\right)
$$

and $s_{1} s_{2} \in S$ while $\left(r_{i} s_{2}+s_{1} r_{i-1}+r_{i} r_{i-1}\right) \in R^{i}$.

Let $A_{\lambda}, \lambda \in \Lambda$, be a collection of subsets of a ring $R$. We shall denote by $R g\left\langle\mathrm{~A}_{\lambda} \mid \lambda \in \Lambda\right\rangle$ the smallest subring of $R$ which contains the set theoretic union of the $A_{\lambda}$ 's. If $R=R g\left\langle A_{\lambda} \mid \lambda \in \Lambda\right\rangle$ we say that $R$ is generated by $A_{\lambda}, \lambda \in \Lambda$.

We shall use the notation $\mathscr{B}_{0}$ for the class of rings which are generated by their $\mathscr{A}$-subideals. We shall prove later that the class of rings which are generated by their $\mathscr{N}$-subideals coincides with the class $\mathscr{F}$.

A subring $S$ of a ring $R$ is a meta ideal of $R$ if there exist subrings $S_{\lambda}$ of $R$ with

$$
S=S_{1} \subseteq S_{2} \subseteq \ldots \subseteq S_{\lambda} \subseteq \ldots S_{\mu}=R,
$$

where $S_{\lambda}$ is an ideal of $S_{\lambda+1}$ for all $\lambda<\mu$ and if $\lambda$ is a limit ordinal $S_{\lambda}=\bigcup_{\alpha<\lambda} S_{\alpha}$. 
We shall use the notation $\mathscr{G}_{0}$ and $\mathscr{G}_{1}$ for the classes of rings which are generated by their $\mathscr{A}$-meta ideals and their $\mathscr{N}$-meta ideals respectively. Finally we shall define the class $\mathscr{Z}$ by: $R \in \mathscr{Z}$ if and only if $R$ has an ascending chain of ideals

$$
(0)=A_{1} \subseteq A_{2} \subseteq \ldots \subseteq A_{\lambda} \subseteq \ldots \subseteq A_{\mu}=R,
$$

where $R A_{\lambda+1} \subseteq A_{\lambda}$ for all $\lambda<\mu$ and if $\lambda$ is a limit ordinal $A_{\lambda}=\bigcup_{\alpha<\lambda} A_{\alpha}$. The class of locally nilpotent rings, that is rings in which every finite set of elements is contained in a nilpotent subring, we shall denote by $\mathscr{L} \mathscr{N}$.

\section{Inclusions between classes}

We shall prove in this section that the inclusion relations between the classes we have defined are as given by the following lattice diagram. This diagram shows only strict inclusions between the classes and there are no inclusions other than those shown.

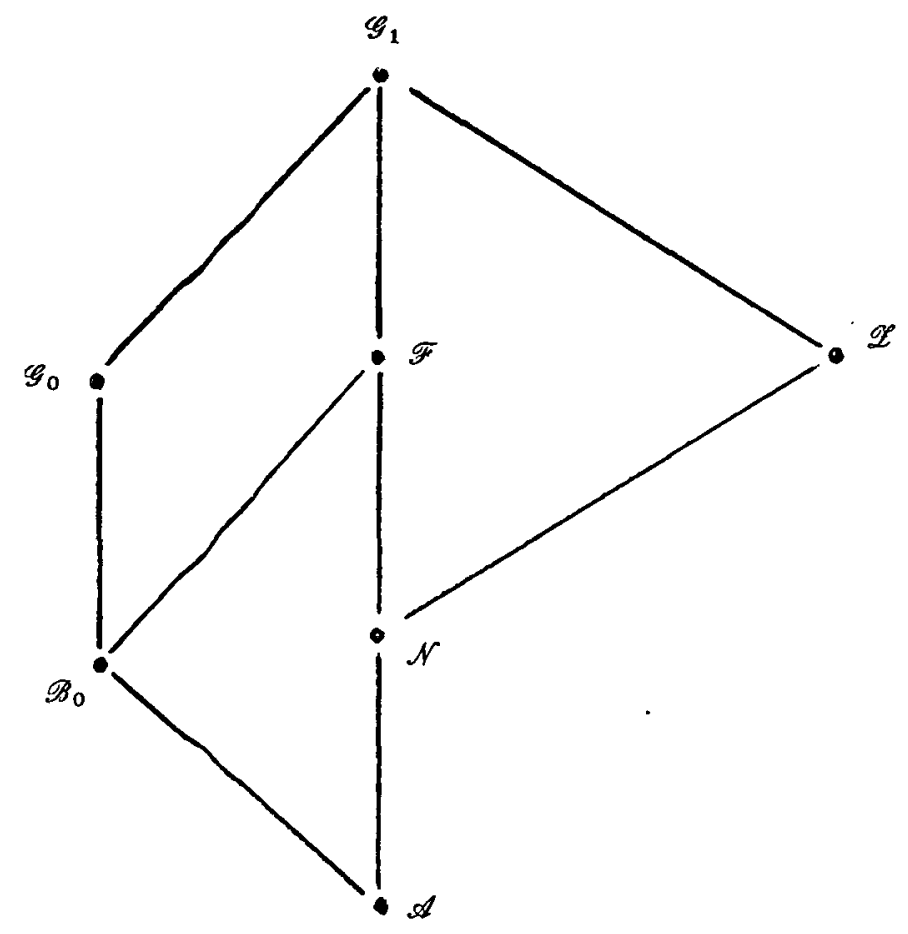

All these classes are contained in the class $\mathscr{L} \mathcal{N}$.

These inclusions are all obvious except perhaps for $\mathscr{B}_{0} \subseteq \mathscr{F}, \mathscr{Z} \subseteq \mathscr{G}_{1}$ and $\mathscr{G}_{1} \subseteq \mathscr{L} \mathscr{N}$. See (4) page 4 for a characterisation of nilpotent rings which gives $\mathscr{N} \subseteq \mathscr{Z}$. 
Theorem 1. The ideal of a ring generated by a nilpotent subideal is nilpotent.

Proof. Let $I$ be a subideal of a ring $R$. Then

$$
I=A_{0} \subseteq A_{1} \subseteq \ldots \subseteq A_{n}=R,
$$

where $A_{i-1}$ is an ideal of $A_{i}, 1 \leqq i \leqq n$. Call $I$ an $n$-step subideal. Let $I$ be the ideal of $R$ generated by $I$. We shall show that $I^{3^{n}} \subseteq I$. We use induction on $n$. If $J$ is the ideal of $A_{2}$ generated by $I$, then

$$
J \subseteq A_{2} \subseteq A_{3} \subseteq \ldots \subseteq A_{n}=R,
$$

and $J$ is an $n-1$ step subideal. Hence by induction, $\bar{J}^{3^{n-1}} \subseteq J$, where $\bar{J}$ is the ideal of $R$ generated by $J$. But then since $I \subseteq A_{1} \subseteq A_{2}$ and $J$ is the ideal of $A_{2}$ generated by $I, J^{3} \subseteq I$, by Andrunakievic's Lemma. Then $\bar{J}^{3^{n}} \subseteq J^{3} \subseteq I$. However $I \subseteq J \subseteq \bar{I}$ so $\bar{J}=\bar{I}$ giving $\bar{I}^{3^{n}} \subseteq I$. Therefore, if $I^{m}=0$, we have $\left(\bar{I}^{3^{n}}\right)^{m}=0$ so $\vec{I}^{3^{n_{m}}}=0$, proving that $\bar{I}$ is nilpotent.

Corollary. $R \in \mathscr{F}$ if and only if $R$ is generated by its $\mathcal{N}$-subideals.

Proof. If $R \in \mathscr{F}$ then it is clear that $R$ is generated by its $\mathscr{N}$-subideals.

Conversely, suppose $R$ is generated by its $\mathscr{N}$-subideals. Then

$$
\left.R=R g\left\langle N_{\lambda}\right| \lambda \in \Lambda, N_{\lambda} \in \mathcal{N} \text { and } N_{\lambda} \text { a subideal of } R\right\rangle .
$$

But by the theorem $\bar{N}_{\lambda} \in \mathscr{N}$ for all $\lambda \in \Lambda$ and so

$$
\left.R=R g\left\langle\bar{N}_{\lambda}\right| \lambda \in \Lambda, \bar{N}_{\lambda} \in \mathcal{N}, \bar{N}_{\lambda} \text { an ideal of } R\right\rangle .
$$

Hence $R$ is the sum of its nilpotent ideals; so $R \in \mathscr{F}$.

It now follows immediately that

Corollary. $\mathscr{B}_{0} \subseteq \mathscr{F}$.

Theorem 2. The ideal of a ring generated by a locally nilpotent meta ideal is locally nilpotent.

Proof. Let $S$ be a meta ideal of a ring $R$. Then

$$
S=A_{0} \subseteq A_{1} \subseteq \ldots \subseteq A_{\lambda} \subseteq \ldots \subseteq A_{\mu}=R,
$$

where $A_{\lambda}$ is an ideal of $A_{\lambda+1}$ for $\lambda<\mu$ and for limit ordinals $\lambda, A_{\lambda}=\bigcup_{\alpha<\lambda} A_{\alpha}$. Now let $M_{\lambda}$ be the ideal of $A_{\lambda}$ generated by $S$. Then

$$
S=M_{1} \subseteq M_{2} \subseteq \ldots \subseteq M_{\lambda} \subseteq \ldots \subseteq M_{\mu} \subseteq R
$$

Now $M_{\lambda+1} \subseteq A_{\lambda} \subseteq A_{\lambda+1}$ since $M_{\lambda+1}$ is the smallest ideal of $A_{\lambda+1}$ containing $S$. Since $M_{\lambda} \subseteq M_{\lambda+1}$ we get $M_{\lambda}$ an ideal of $M_{\lambda+1}$.

We must show that $M_{\mu}$ is locally nilpotent. Suppose not. Then since $S$ is locally nilpotent there is a first $\lambda$ with $M_{\lambda}$ not locally nilpotent. Now $\lambda$ is not a limit ordinal for then $M_{\lambda}=\bigcup_{\alpha<\lambda} M_{\alpha}$ and $M_{a}, \alpha<\lambda$, is locally nilpotent. This gives $M_{\lambda}$ is locally nilpotent which is a contradiction. Now if $\lambda$ is not a limit 
ordinal $\lambda-1$ exists and $M_{\lambda-1}$ exists and $M_{\lambda-1}$ is an ideal of $M_{2}$ which is itself an ideal of $A_{\lambda}$. So if $J$ is the ideal generated by $M_{\lambda-1}$ in $A_{\lambda}$ we have

$$
J^{3} \subseteq M_{\lambda-1} .
$$

But $M_{\lambda}$ is the ideal generated by $S$ in $A_{\lambda}$ so $M_{\lambda} \subseteq J$. Hence $M_{\lambda}^{3} \subseteq M_{\lambda-1}$. But $M_{\lambda-1}$ is locally nilpotent so $M_{\lambda}^{3}$ is locally nilpotent. Then if $X$ is any finitely generated subring of $M_{\lambda}$ we have

$$
X^{3} \subseteq M_{\lambda}^{3}, X^{3} \text { finitely generated. }
$$

But then $X^{3}$ is nilpotent, say $\left(X^{3}\right)^{m}=(0)$. This shows that $X$ is nilpotent and so $M_{\lambda}$ is locally nilpotent giving a contradiction.

Corollary. $\mathscr{G}_{1} \subseteq \mathscr{L} \mathcal{N}$.

Proof. Let $R \in \mathscr{G}_{1}$. Then

$$
\left.R=R g\left\langle N_{\lambda}\right| \lambda \in \Lambda, N_{\lambda} \in \mathcal{N}, N_{\lambda} \text { a meta ideal of } R\right\rangle .
$$

But then $\bar{N}_{\lambda}$, the ideal generated by $N_{\lambda}$ is in $\mathscr{L} \mathcal{N}$. Thus

$$
\left.R=R g\left\langle\bar{N}_{\lambda}\right| \lambda \in \Lambda, \bar{N}_{\lambda} \in \mathscr{L} \mathscr{N}, \bar{N}_{\lambda} \text { an ideal of } R\right\rangle .
$$

Hence $R=\sum_{\lambda \in \Lambda} \bar{N}_{\lambda}$, giving $R \in \mathscr{L} \mathscr{N}$.

A ring in which every subring is a meta ideal is called a $U$-ring. In (2) Freidman proved that every nil $U$-ring is locally nilpotent and since a nil $U$-ring is clearly in $\mathscr{G}_{1}$ this result is a special case of the above corollary. It is also worth noting that the class $\mathscr{G}_{1}$ strictly contains the class of nil $U$-rings. For, consider the vector space $R$ over $G F(p), p$ a prime, with basis the set of symbols

$$
\left\{a_{\lambda} \mid \lambda \text { a rational, } 0<\lambda<1\right\} \text {. }
$$

Now $R$ can be made into an algebra over $G F(p)$ by defining multiplication on the basis elements by

$$
\begin{aligned}
a_{\lambda} a_{\mu} & =a_{\lambda+\mu} \text { if } \lambda+\mu<1 . \\
& =0 \text { if } \lambda+\mu \geqq 1 .
\end{aligned}
$$

Then $R$ is an $\mathscr{F}$-ring since $A_{\lambda}$, the ideal generated by $a_{\lambda}$, is nilpotent and $R=\sum_{\lambda \in(0,1)} A_{\lambda}$. Hence $R \in \mathscr{G}_{1}$ but $R$ is not a $U$-ring; see (1) for a proof of this result.

The next result shows that every $\mathscr{Z}$-ring is a nil $U$-ring.

Theorem 3. Every subring of a $\mathscr{Z}$-ring is a meta ideal.

Proof. Let $R \in \mathscr{Z}$. Then we have a chain of ideals

$$
\text { (0) }=A_{1} \subseteq A_{2} \subseteq \ldots \subseteq A_{\lambda} \subseteq \ldots \subseteq A_{\mu}=R,
$$

with $R A_{\lambda+1} \subseteq A_{\lambda}$ for $\lambda<\mu$ and $A_{\lambda}=\bigcup_{\alpha<\lambda} A_{\alpha}$ for limit ordinals $\lambda$. Suppose $S$ is a subring of $R$. Then define $S_{\lambda}=S+A_{\lambda}$. We have

$$
S=S_{1} \subseteq S_{2} \subseteq \ldots \subseteq S_{\lambda} \subseteq \ldots \subseteq S_{\mu}=R \text {. }
$$


Now $S_{\lambda}$ is an ideal of $S_{\lambda+1}$. For, given $s+a \in S+A_{\lambda}$ and $s_{1}+a_{1} \in S+A_{\lambda+1}$ then

$$
(s+a)\left(s_{1}+a_{1}\right)=s s_{1}+\left(a s_{1}+s a_{1}+a a_{1}\right) .
$$

But $s s_{1} \in S$ and $\left(a s_{1}+s a_{1}+a a_{1}\right) \in A_{\lambda}$; so we have the result.

Corollary. $\mathscr{Z} \subseteq \mathscr{G}_{1}$.

Proof. Clearly it will be sufficient to prove that if $R \in \mathscr{Z}$ then $R$ is a nil ring. For then, by the theorem, $R \in \mathscr{G}_{1}$ since every element will generate a nilpotent subring which must be a meta ideal.

Let $a \in R$. Consider $T=\left\{a, a^{2}, a^{3}, \ldots\right\}$. If $a^{n} \neq 0$ for any $n \geqq 1$ then there is a first $A_{\alpha}$ with $A_{\alpha} \cap T=\varnothing$ while $A_{\alpha+1} \cap T \neq \varnothing$. Let $a^{n} \in A_{\alpha+1}$. Since $R A_{\alpha+1} \subseteq A_{\alpha}$ we have $a a^{n} \in A_{\alpha}$ so $\operatorname{T\cap } A_{\alpha} \neq \varnothing$, which is a contradiction. Hence $a$ is nilpotent.

\section{Examples}

To show that the inclusions in the lattice diagram of Section 2 are proper we look at subrings of infinite dimensional matrices. Let $\Omega$ be any totally ordered set and $R$ an arbitrary ring. Let $M_{\Omega}(R)$ denote the matrix ring of $\Omega \times \Omega$ matrices over $R$, the matrices differing from the zero matrix in at most a finite number of places. We shall look first at the subring, $R_{\Omega}(F)$, of upper zero triangular matrices of $M_{\Omega}(F)$, where $F$ is a field. We shall use the notation $x e_{\lambda \mu}$ for the matrix in $M_{\Omega}(R)$ whose only non-zero entry is $x \in R$ in the $(\lambda, \mu)$ th position.

Theorem 4. $R_{\Omega}(F) \in \mathscr{B}_{0}$ for any field $F$ and any totally ordered set $\Omega$.

Proof. Let $x e_{\lambda \mu} \in R_{\Omega}(F)$, where $x \in F, \lambda, \mu \in \Omega$ with $\lambda<\mu$. Let $E_{\lambda \mu}$ denote the ideal generated by $x e_{\lambda \mu}$ in $R_{\Omega}(F)$. Now

$$
\left(x e_{\lambda \mu}\right) e_{\mu \beta} \in E_{\lambda \mu} \text { for any } \beta>\mu \text {. }
$$

Hence $x e_{\lambda \beta} \in E_{\lambda \mu}$ for $\beta \geqq \mu$. Also

$$
\left(y x^{-1} e_{\alpha \lambda}\right)\left(x e_{\lambda \beta}\right) \in E_{\lambda \mu} \text { for any } \alpha<\lambda \text { and } y \in F \text {. }
$$

Hence $y e_{\alpha \beta} \in E_{\lambda \mu}$ for any $\alpha \leqq \lambda<\mu \leqq \beta$ and $y \in F$. But then clearly we have

$$
E_{\lambda \mu}=R g\left\langle y e_{\alpha \beta} \mid \alpha \leqq \lambda<\mu \leqq \beta, y \in F\right\rangle,
$$

since this subring is an ideal of $R_{\Omega}(F)$.

However, then $R g\left\langle x e_{\lambda \mu}\right\rangle$ is an ideal of $E_{\lambda \mu}$ which itself is an ideal of $R_{\Omega}(F)$. Since $R g\left\langle x e_{\lambda \mu}\right\rangle \in \mathscr{A}$ this shows that $R_{\Omega}(F)$ is generated by its $\mathscr{A}$-subideals. Hence $R_{\mathbf{\Omega}}(F) \in \mathscr{B}_{0}$.

Now if $\Omega$ fails to possess either a greatest or a least element, $R_{\Omega}(F)$ has trivial annihilator. For, suppose

$$
A=\operatorname{ann} R_{\Omega}(F) \text { and } \sum_{i=1}^{n} x_{i} e_{\lambda_{i} \mu_{i}} \in A \text { with } \sum_{i=1}^{n} x_{i} e_{\lambda_{i} \mu_{i}} \neq 0 .
$$


If $\Omega$ has no greatest element choose $\rho>\mu_{i}, i=1, \ldots, n$. Then

$$
\left(\sum_{i=1}^{n} x_{i} e_{\lambda_{i} \mu_{i}}\right) e_{\mu_{1} \rho}=\sum_{j \in J} x_{j} e_{\lambda_{j} \rho} \neq 0
$$

where $J=\left\{j \mid \mu_{j}=\mu_{i}, 1 \leqq j \leqq n\right\}$.

Similarly, if $\Omega$ has no least element we can choose $\sigma<\lambda_{i}, 1 \leqq i \leqq n$, and then

$$
e_{\sigma \lambda_{1}}\left(\sum_{i=1}^{n} x_{i} e_{\lambda_{i}} \mu_{i}\right) \neq 0
$$

We now get immediately the following result.

Lemma 5. If $\Omega$ is infinite then $R_{\Omega}(F) \notin \mathscr{Z}$.

Proof. Since $\Omega$ is infinite we can choose a subset $\Lambda$ of $\Omega$, the set $\Lambda$ either failing to possess a greatest element or failing to possess a least element. Then $R_{\Lambda}(F) \notin \mathscr{Z}$ and since $R_{\Lambda}(F) \leqq R_{\Omega}(F)$ and a subring of a $\mathscr{Z}$-ring is a $\mathscr{Z}$-ring this gives $R_{\mathbf{\Omega}}(F) \notin \mathscr{Z}$.

. Hence if $\Omega$ is infinite and $F$ is any field, $R_{\Omega}(F) \in \mathscr{B}_{0}$ but $R_{\Omega}(F) \notin \mathscr{N}$. Also $R_{\Omega}(F) \in \mathscr{G}_{0}$ but $R_{\Omega}(F) \notin \mathscr{Z}$. To see that $\mathscr{N} \neq \mathscr{Z}$ notice that for any field $F$

$$
\bigoplus_{n=1}^{\infty} R_{n}(F) \in \mathscr{Z} \text { but } \bigoplus_{n=1}^{\infty} R_{n}(F) \notin \mathscr{N}
$$

since $R_{n}(F)$ is nilpotent of class $n$.

The only other inclusions that it is not trivial to show proper are $\mathscr{B}_{0} \subset \mathscr{G}_{0}$ and $\mathscr{F} \subset \mathscr{G}_{1}$. The next results are aimed at proving that these are in fact the case.

Theorem 6. If $\Omega$ is a densely ordered set then $\left(R_{\Omega}(F)\right)^{2}=R_{\Omega}(F)$.

Proof. $R_{\Omega}(F)$ is generated by elements of the form $a e_{\lambda \mu}, a \in F, \lambda, \mu \in \Omega$ with $\lambda<\mu$. Since $\Omega$ is densely ordered there is an element $\rho \in \Omega$ with $\lambda<\rho<\mu$. Then

$$
a e_{\lambda \mu}=\left(a e_{\lambda \rho}\right) e_{\rho \mu} \in\left(R_{\Omega}(F)\right)^{2}
$$

The result follows.

Suppose $R$ is any ring and $A$ is an ideal of $R$. For $\Omega$ a totally ordered set define $M_{\Omega}(R, A)$ to be the subring of $M_{\Omega}(R)$ consisting of those matrices of $M_{\Omega}(R)$ which are upper zero triangular when the entries are taken modulo $A$. Then

$$
M_{\Omega}(R, R)=M_{\Omega}(R) \text { and } M_{\Omega}(R,(0))=R_{\Omega}(R) .
$$

If $R$ is any ring denote by $R^{*}$, the usual ring with a 1 containing $R$ as an ideal, see (3) page 11 .

Theorem 7. If $R \in \mathscr{F}$ then $M_{\Omega}\left(R^{*}, R\right) \in \mathscr{G}_{1}$. 
Proof. Let $R \in \mathscr{F}$. To show $M_{\Omega}\left(R^{*}, R\right) \in \mathscr{G}_{1}$ we show first that

$$
H=H_{\lambda \mu}\left(r^{*}\right)=R g\left\langle M_{\Omega}(R), r e_{\lambda \mu}\right\rangle \in \mathscr{G}_{1},
$$

where $r^{*}$ is a fixed element of $R^{*}$ and $\lambda, \mu$ are fixed elements of $\Omega$ with $\lambda<\mu$. Since $H$ is a subideal of $M_{\Omega}\left(R^{*}, R\right)$ this will show that the $\mathscr{N}$-meta ideals of $H$ are $\mathcal{N}$-meta ideals of $M_{\Omega}\left(R^{*}, R\right)$. However, $M_{\Omega}\left(R^{*}, R\right)$ is generated by subrings $H_{\lambda \mu}\left(r^{*}\right)$ as $\lambda, \mu$ vary through $\Omega$ with $\lambda<\mu$ and $r^{*}$ varies through $R^{*}$. This gives $M_{\Omega}\left(R^{*}, R\right) \in \mathscr{G}_{1}$ as required.

To show $H \in \mathscr{G}_{1}$ it is sufficient to prove that $R g\left\langle r^{*} e_{\lambda \mu}\right\rangle$ is a meta ideal of $H$. For certainly $M_{\Omega}(R)$ is an ideal of $H$ and is generated by $\mathscr{N}$-ideals since $R$ is the sum of its $\mathscr{N}$-ideals. Hence $H$ will be generated by the $\mathscr{N}$-subideals which generate $M_{\Omega}(R)$ and the $\mathscr{A}$-meta ideal $R g\left\langle r^{*} e_{\lambda \mu}\right\rangle$.

Since $R \in \mathscr{F}, R$ has a chain of ideals

$$
(0)=M_{0} \subseteq M_{1} \subseteq \ldots \subseteq M_{t} \subseteq \ldots \subseteq M_{s}=R,
$$

with $M_{t+1}^{2} \subseteq M_{t}$ for all $t<s$ and, for limit ordinals $t, M_{t}=\bigcup_{x<t} M_{x}$, because it is well known that an $\mathscr{F}$-ring is a Baer lower radical ring.

To prove $R g\left\langle r^{*} e_{\lambda \mu}\right\rangle$ is a meta ideal of $H$ define

for a non-limit ordinal $t$,

$$
H_{0}=R g\left\langle r^{*} e_{\lambda \mu}\right\rangle
$$

$$
\begin{aligned}
H_{3 t} & =R g\left\langle H_{3 t-1}, r e_{\alpha \beta} \mid \beta \neq \lambda, \alpha \neq \mu, r \in M_{t}\right\rangle, \\
H_{3 t+1} & =R g\left\langle H_{3 t}, r e_{\lambda \lambda}, r e_{\mu \mu} \mid r \in M_{t}\right\rangle, \\
H_{3 t+2} & =R g\left\langle H_{3 t+1}, r e_{\mu \rho}, r e_{\rho \lambda} \mid r \in M_{t}\right\rangle,
\end{aligned}
$$

and for limit ordinals $t$ define

$$
H_{t}=\bigcup_{x<t} H_{x}
$$

Then for any ordinal $t$ we have $H_{t}$ an ideal of $H_{t+1}$ and $H=\bigcup_{t} H_{t}$. The result follows.

Theorem 8. If $\Omega$ is infinite and $R$ is a ring with $R^{2}=R$ then $M_{\Omega}\left(R^{*}, R\right) \notin \mathscr{F}$.

Proof. Let $S$ be the subring of $M_{\Omega}\left(R^{*}, R\right)$ defined by

$$
\left.S=R g\left\langle e_{\lambda \mu}, r e_{\alpha \beta}\right| r \in R, \lambda<\mu, \lambda, \mu \text { fixed }\right\rangle .
$$

Then it is sufficient to show that $S \notin \mathscr{F}$.

Let $T$ be the ideal of $S$ generated by $e_{\lambda \mu}$. Then given any $\alpha, \beta \in \Omega$ choose $\gamma$, $\delta \in \Omega$ with $\alpha, \beta \neq \gamma, \delta$ and $\gamma, \delta \neq \lambda, \mu$. Then given $r \in R$ we have

But this gives

$$
r=\sum_{i=1}^{n} r_{1 i} r_{2 i}
$$

$$
r e_{\gamma \delta}=\sum_{i=1}^{n} r_{1 i} e_{\gamma \alpha} e_{\alpha \beta} r_{2 i} e_{\beta \delta} \in T .
$$


Similarly $r e_{\alpha \beta} \in T$ and so $T=S$. But $S \notin \mathscr{N}$ for $\Omega$ infinite since $R_{\Omega}\left(R^{*}\right) \notin \mathscr{N}$. Hence $M_{\mathbf{\Omega}}\left(R^{*}, R\right) \notin \mathscr{F}$.

As a corollary to Theorems 7 and 8 we have

Corollary. $\mathscr{F} \neq \mathscr{G}_{1}$.

A straightforward modification of the proof of Theorem 7 gives

Theorem 9. If $R \in \mathscr{B}_{0}$ then $M_{\Omega}\left(R^{*}, R\right) \in \mathscr{G}_{0}$.

From Theorems 4 and 6 , if $\Omega$ is densely ordered and $F$ is any field,

$$
R_{\Omega}(F) \in \mathscr{B}_{0} \text { and } R_{\Omega}(F)^{2}=R_{\Omega}(F) \text {. }
$$

Hence for any infinite totally ordered set $\Lambda$,

But, by Theorem 8 ,

$$
M_{\Lambda}\left(R_{\Omega}(F)^{*}, R_{\Omega}(F)\right) \in \mathscr{G}_{0} .
$$

$$
M_{\Lambda}\left(R_{\Omega}(F)^{*}, R_{\Omega}(F)\right) \notin \mathscr{F} .
$$

Since $\mathscr{B}_{0} \leqq \mathscr{F}$ this shows that $\mathscr{B}_{0} \neq \mathscr{G}_{0}$ as required.

Finally we give examples to show $\mathscr{N} \ddagger \mathscr{G}_{0}$ and $\mathscr{Z} \ddagger \mathscr{F}$ to complete the proof that there are no hidden inclusions in the lattice inclusion diagram.

The ring $2 \mathbf{Z} / 8 \mathbf{Z}$ provides an example that $\mathscr{N} \ddagger \mathscr{G}_{0}$.

To show $\mathscr{Z} \notin \mathscr{F}$ let $R=\bigoplus_{n=1}^{\infty} R_{n}(F)$. We have shown that $R \in \mathscr{Z}$ and it is straightforward to check that $M_{2}\left(R^{*}, R\right) \in \mathscr{Z}$ but $M_{2}\left(R^{*}, R\right) \notin \mathscr{F}$.

\section{REFERENCES}

(1) R. G. Biggs, A new bound for nil $U$-rings, Canad. J. Math. 22 (1970), 403-407.

(2) P. A. Friedman, Rings with an idealizer condition, Izv. Vysš Učebn. Zaved. Mathematika, 15 (1960), 213-222 (Russian).

(3) N. JACOBSON, Structure of Rings (Amer. Math. Soc. Colloq. Publ. XXXVII). 1969).

(4) R. L. KruSE and D. T. PrICE, Nilpotent Rings (Gordon and Breach, New York, 300.

(5) E. F. Robertson, On certain subgroups of $G L(R)$, J. Algebra 15 (1970), 293-

(6) E. F. Robertson, Some properties of a subgroup of $S p_{\Omega}(R), J$. London Math. Soc. (2), 4 (1971), 65-78.

Mathematical Institute

NoRTH HAUgh

ST ANDREWS 